\title{
Gender diversity management, culture inclusive e sfide dell'at- tualità. Una review sistematica della letteratura
}

\author{
Looking Back and Looking Forward: Equity challenges and \\ Gender Diversity Management. A literature Review \\ Alessandra Romano*, Rubina Petruccioli**
}

\begin{abstract}
Riassunto
L'articolo esplora i dispositivi di gender equity e le azioni per il sostegno alle carriere femminili a partire dalla review sistematica degli studi empirici sul tema dell'ultimo ventennio (2000-2020). Sono stati analizzati centodieci contributi nazionali e internazionali. Le finalità della review sono contribuire a ricostruire lo stato dell'arte degli studi nazionali e internazionali su gender equity nei workplace, segregazioni di genere e work-life balance e offrire un quadro di sintesi rispetto alle proposte di intervento e ai dispositivi di supporto alle carriere femminili.

L'obiettivo è individuare traiettorie di intervento utili a sviluppare modelli culturali ed educativi validi per coltivare processi di inclusione di genere nelle organizzazioni e nei contesti di lavoro.
\end{abstract}

Parole chiave: parità di genere, barriere, work-life balance, carriere femminili, segregazioni.

\section{Abstract}

In an effort to provide a more in-depth understanding of essential components of gender equity and actions for gender diversity management, the purpose of this article is to review the empirical literature in the last two decades (20002020) that focuses on gender equity strategies in workplaces. One-hundred and ten empirical studies were reviewed that foreground gender equity and

\footnotetext{
* Ricercatrice Senior in Didattica e pedagogia speciale presso l'Università degli Studi di Siena. E-mail: alessandra.romano2@unisi.it.

${ }^{* *}$ Dipartimento di Scienze della formazione, scienze umane e della comunicazione interculturale, Università degli Studi di Siena.

Il lavoro di review e di stesura del contributo è frutto di continui scambi e riflessioni tra le due Autrici. Solo per ragioni di responsabilità scientifica, si specifica che i paragrafi 1., 2., 3. (3.1., 3.2., 3.3., 3.4.1.), 4. sono da attribuire ad Alessandra Romano, mentre le sezioni 3.4.2., 3.4.3. e 3.4.4. e l'editing grafico sono da attribuire a Rubina Petruccioli.
}

Doi: $10.3280 /$ ess 1-2020oa9477 
management strategies. Findings identified four clusters of issues related to gender equity in management position, gender representatives, work-life balance and actions for supporting female careers development.

A detailed agenda for future research is outlined, building on the gaps identified by the review and founded on renewed theoretical direction.

Keywords: gender equity, barriers, work-life balance, female careers, segregations.

\section{La diversità di genere nelle organizzazioni}

L'articolo esplora i dispositivi di gender equity e le azioni per il sostegno alle carriere femminili a partire dalla ricostruzione della letteratura degli studi empirici sul tema.

Quali sono le traiettorie attuali? Quali traiettorie future potremmo percorrere? Qual è il contributo dei modelli educativi per la diffusione di una cultura che sia gender-friendly e gender-sensitive?

Sappiamo che nei paesi Ocse, in media, per ogni donna manager ci sono due uomini che ricoprono lo stesso ruolo: in Germania, ad esempio, la percentuale di uomini con una posizione dirigenziale sul totale della forza lavoro è pari al $6 \%$, mentre le donne sono il $2,8 \%$; in Francia, la quota maschile è del 9,3\%, quella femminile del 4,6\%; nel Regno Unito $13 \%$ a fronte dell' $8,1 \%$; nei Paesi Bassi $8,4 \%$ a fronte del 3,5\%. L'Italia mostra un profilo coerente con le tendenze generali: nel nostro paese, infatti, i manager uomini rappresentano il $4,7 \%$ della forza lavoro, mentre le donne sono il 2,4\% (Carbone, \& Dagnes, $2019)^{1}$. Le trasformazioni avvenute a livello sociale hanno inciso sulla partecipazione femminile al mercato del lavoro in termini quantitativi, aumentandone la presenza, ma anche qualitativi, in quanto le donne hanno avuto accesso a settori ad appannaggio esclusivamente maschili. L'ampliamento, però, si è tradotto in un'espansione pubblica di compiti e mandati svolti all'interno della sfera privata, riproducendo script interazionali a impostazione fortemente sessista anche all'interno di contesti organizzativi. Come arginare tendenze riproduttive di una visione svalutante e sottostimante delle carriere femminili in posizioni di governance? Queste sono sfide educative che richiedono di sovvertire l'ordine simbolico di potere, di ripensare le azioni di sostegno alle carriere di genere, di validare i modelli di intervento per il work life balance, di contrastare

${ }^{1}$ Dati Ocse 2015. 
le segregazioni occupazionali di genere, sia verticali che orizzontali (Blackburn et al., 2002). Richiamano alcune riflessioni più generali circa il rapporto tra partecipazione femminile al mercato del lavoro, equità di genere e accesso alle posizioni apicali, inclusione nelle imprese, scenari incerti e precarietà professionali del terzo millennio. Il focus è sul supportare processi di validazione di bias e stereotipi vecchi e nuovi all'interno delle culture organizzative e facilitare lo sviluppo delle carriere femminili a fronte di discriminazioni, svalutazioni, ostacoli e barriere.

Approfondire i dispositivi e le azioni a sostegno della gender equity nei workplace risponde sia a un bisogno conoscitivo, comprendere lo stato dell'arte dei contributi sulla gender equity in modo scientificamente validato, sia a un intento critico-emancipativo, ricollocare le questioni della gender equity all'interno dello studio di processi di cambiamento organizzativo e di trasformazione delle pratiche sessiste ancora in uso.

In questo scenario ci proponiamo di offrire un quadro - seppur limitato della letteratura che si occupa dei dispositivi a sostegno della gender equity e delle azioni che sono state messe in atto per contrastare i fenomeni di segregazione delle carriere femminili. Si tratta di ricerche condotte nell'ambito degli studi organizzativi, degli studi manageriali, degli studi di genere e degli studi sullo sviluppo professionale. Gli studi manageriali e i contributi dell'apprendimento organizzativo ci restituiscono che la progettazione e la realizzazione di azioni a supporto delle politiche di gender equity sono una questione di cambiamento organizzativo e di sostenibilità di genere come asset strategico per l'innovazione. Il nostro intento è individuare traiettorie di intervento utili a supportare modelli culturali ed educativi validi per il presente e atti a ridisegnare un futuro sempre più inclusivo e gender-sensitive.

\section{Inostri ancoraggi teorici}

Effettuare una review della letteratura non è mai un compito facile: avevamo di fronte un corpus decisamente vasto di produzioni scientifiche che affrontavano, in molti casi trasversalmente oppure in modo marginale, l'oggetto di cui intendevamo occuparci. Come costruire la nostra review? Quali riferimenti concettuali e metodologici avevamo?

Il framework a cui abbiamo fatto riferimento comprendeva i contributi della metodologia della ricerca sulla review della letteratura, offerti da Creswell (2015) e da Feak e Swales (2009). Abbiamo cercato esempi a cui poterci rifare, come la review della letteratura Critical reflection and transformative learning di Taylor (2017), la review sistematica su Arts-based methods in health re- 
search di Fraser e Sayah (2011) e la review transdisciplinare su Maternity management in small and medium-sized enterprises di Stumbitz, Lewis e Rouse (2018).

Abbiamo attinto agli studi sulla letteratura in ambito manageriale e organizzativo sui topic del maternity management, gendered workplaces, familyfriendly working (Stumbitz, Lewis, \& Rouse, 2018; Wang, \& Chugh, 2014). In questi settori, le review sistematiche (Stumbitz, Lewis, \& Rouse, 2018) hanno optato per approcci descrittivi più tradizionali, laddove rispondono a robusti criteri di validità, rigore metodologico e generalizzabilità (Denyer, \& Tranfield, 2009; Jones, \& Gatrell, 2014). Pur riconoscendone i limiti, sono utilizzate soprattutto come "strumenti guida" per studi di natura esplorativa attorno a focus tematici (Stumbitz, Lewis, \& Rouse, 2018).

\section{La metodologia della review}

Le finalità della review sistematica che qui presentiamo sono:

- contribuire a ricostruire lo stato dell'arte degli studi di natura empirica nazionali e internazionali su gender equity nei workplace, segregazioni di genere e work-life balance;

- rilevare le caratteristiche metodologiche delle ricerche in atto su gender equity, segregazioni di genere e work-life balance;

- offrire un quadro di sintesi rispetto alle proposte di intervento e ai dispositivi di supporto alle carriere di genere.

Le domande di ricerca poste sono state:

- quanti studi scientifici sono stati pubblicati su gender equity nei workplace e sostegno alle carriere di genere?

- quali costrutti e procedure di ricerca sono stati adottati?

- quali sono i principali cluster tematici oggetto di indagine?

\subsection{I fase: screening esplorativo attraverso i principali motori di ricerca}

Tra febbraio e marzo 2020 è stato condotto uno screening tematico esplorativo attraverso quattro fasi iterative di ricerca sul database onesearch dell'Università di Siena. I termini chiave utilizzati sono stati:

- gender equity;

- gender diversity strategy;

- female career development;

- gender diversity management;

- work-life balance, woman-friendly, family-friendly,

- segregazione di genere, 
- lavoro femminile,

- donne-lavoro.

I database utilizzati sono stati: Eric, Jstor, OECD library, Sage Journals online, AOM Journals on-line, Springer link, Google Scholar, Rivisteweb, Routledge, Emerald, Wiley.

Le ricerche sono state filtrate includendo articoli peer-reviewed in riviste scientifiche, monografie e volumi collettanei, pubblicati a partire da gennaio 2000. Sono stati esclusi, già nella primissima fase, i prodotti che non fossero pubblicazioni scientifiche nazionali o internazionali e che non rispondessero agli standard di qualità e di accuratezza stabiliti a livello internazionale (Creswell, 2015).

\subsection{Il fase: selezione dei contributi}

Il secondo step è stato quello di revisionare il titolo e l'abstract di 190 articoli internazionali e nazionali, per stabilirne la rilevanza e l'attinenza. In questa fase sono stati esclusi 80 contributi poiché ritenuti non rilevanti ai fini delle domande di ricerca oppure perchè non incontravano $i$ criteri di selezione.

Le due autrici hanno optato per una metodologia di analisi in modo parallelo e indipendente, analizzando singolarmente i contributi, per poi confrontare i risultati delle prime categorizzazioni ai fini della costruzione del corpus della review (Creswell, 2015).

Il contributo, per essere inserito nella selezione, deve:

a) presentare studi, ricerche empiriche o argomentazioni teoriche sulle strategie di gender diversity, gender equity e sullo sviluppo delle carriere femminili;

b) far riferimento alla letteratura sul tema presente negli studi organizzativi, manageriali ed educativi;

c) includere la descrizione di oggetto della ricerca, di partecipanti, di strumenti di indagine e dei principali risultati;

d) presentare dati empirici basati su un approccio di ricerca quantitativo, qualitativo o combinato;

e) offrire riflessioni e proposte di intervento per prevenire, contrastare o ridurre le segregazioni nelle carriere femminili.

Alcuni contributi e saggi rispettavano i criteri di selezione ma non presentavano la descrizione di ricerche condotte first hand quanto meta-analisi oppure mancavano di alcune sezioni di descrizione della metodologia di ricerca. Questi contributi sono stati inclusi nella review poiché ritenuti significativamente rilevanti ai fini dell'oggetto di analisi.

Sono state selezionate restrizioni sulla lingua in cui la pubblicazione è stata scritta, privilegiando pubblicazioni: 
1. in lingua italiana;

2. in lingua inglese.

Abbiamo adottato una logica euristica, inclusiva e transdisciplinare piuttosto che una ricerca su singoli ambiti disciplinari. Complessivamente sono stati selezionati centodieci contributi. Abbiamo escluso dalla review i contributi che affrontano i topic gender equity e gender diversity in Higher Education e Accademia poiché questi avrebbero introdotto nuovi cluster tematici non assoggettabili a quelli individuati sino a quel momento e non rispondenti agli obiettivi della review.

\subsection{III fase: coding e clusterizzazione dei contributi}

Nella terza fase sono stati codificati i contributi, sono stati estratti i dati sulle caratteristiche degli studi e contemporaneamente è stata esaminata la letteratura citata nella lista delle reference e nella bibliografia. Il lavoro di codifica ha prodotto un Code Sheet compilato prima individualmente da ogni ricercatrice e poi confrontato in analisi incrociata.

Nel Code Sheet sono inserite le caratteristiche primarie degli articoli, quali:

- autore/data;

- titolo dell'articolo;

- collocazione geografica;

- oggetto dello studio;

- domande di ricerca;

- metodologia d'indagine (distinguendo l'approccio di ricerca, il disegno di ricerca, gli strumenti di rilevazione dei dati e i partecipanti);

- abstract;

- risultati.

Muovendo dalla composizione del Code Sheet, sono stati individuati i primi cluster tematici che costituivano raggruppamenti di contributi attorno a famiglie di costrutti/problemi.

I cluster tematici sono:

1. gender equity nelle posizioni di management;

2. rappresentanza positiva di genere;

3. work-life balance;

4. azioni a sostegno delle carriere femminili. 
Fig. 1 - Mappa concettuale dei cluster tematici

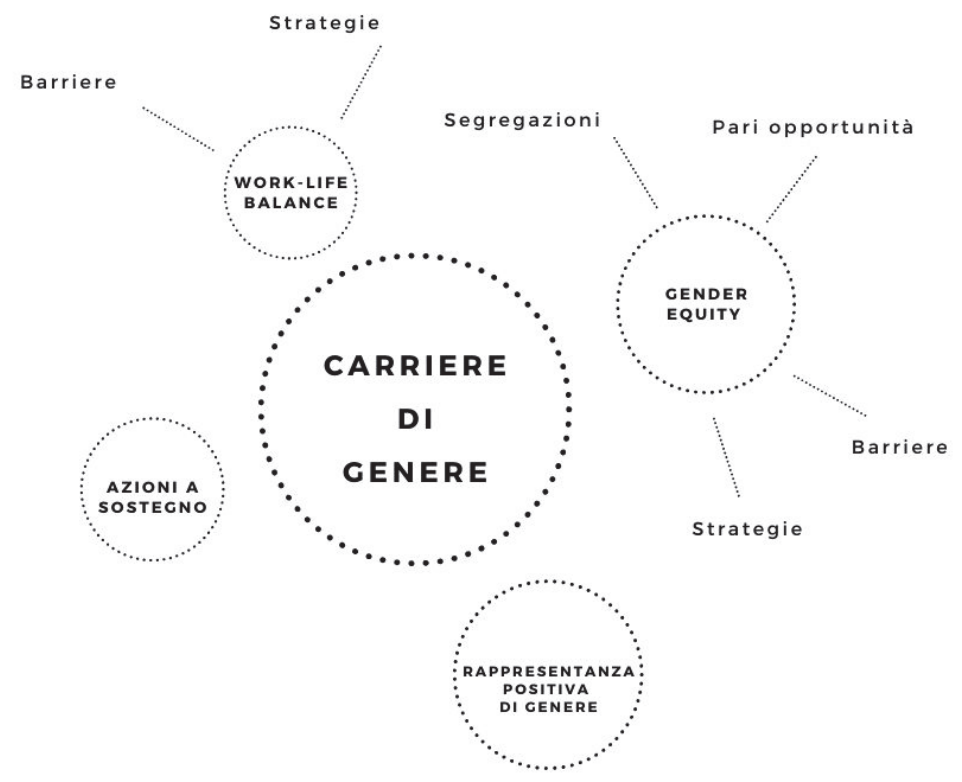


Education Sciences \& Society, 1/2020 ISSN 2038-9442, ISSNe 2284-015X

Fig. 2 - Schema del processo della review della letteratura

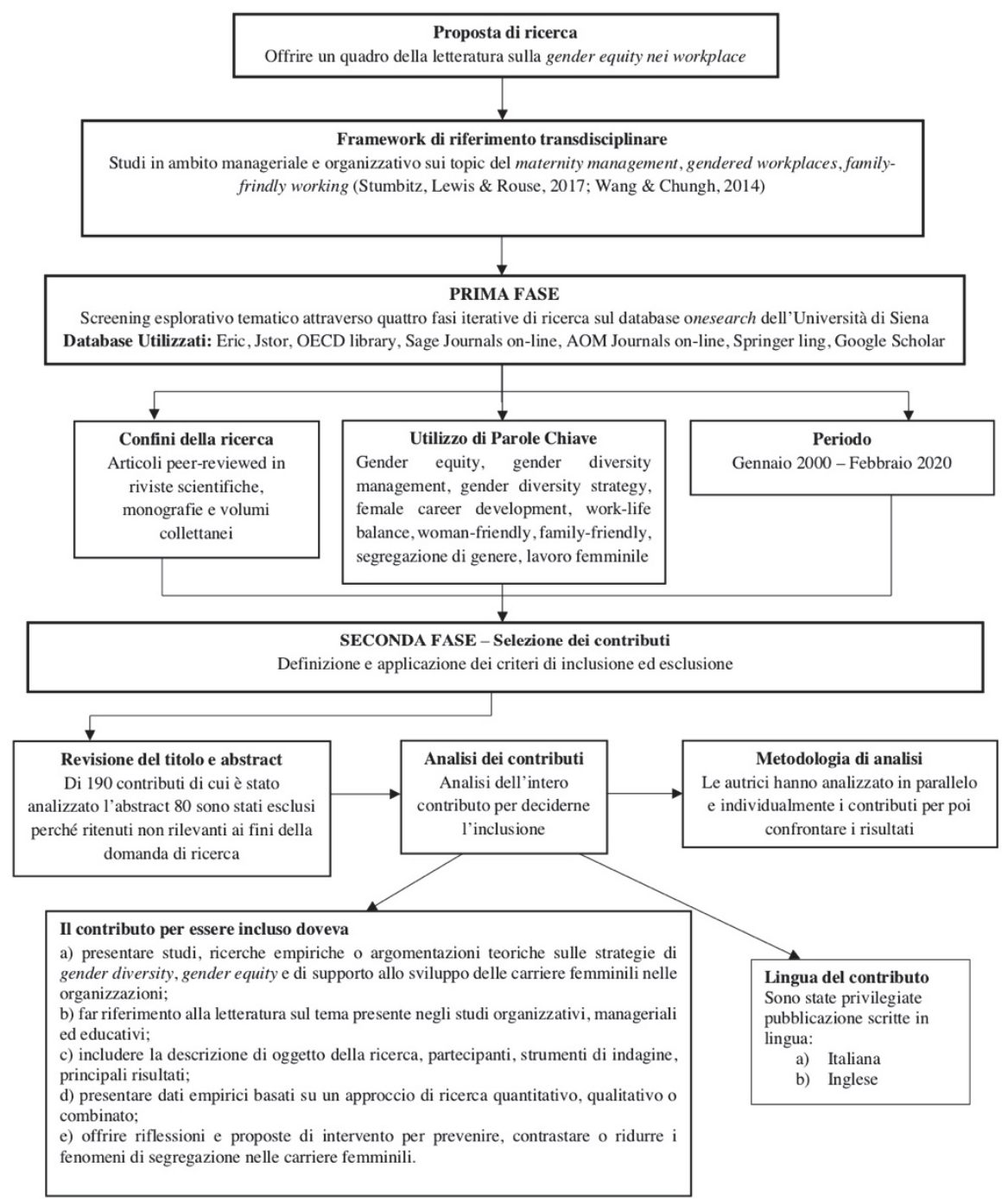


Fig. 2.1 - Schema del processo della review della letteratura

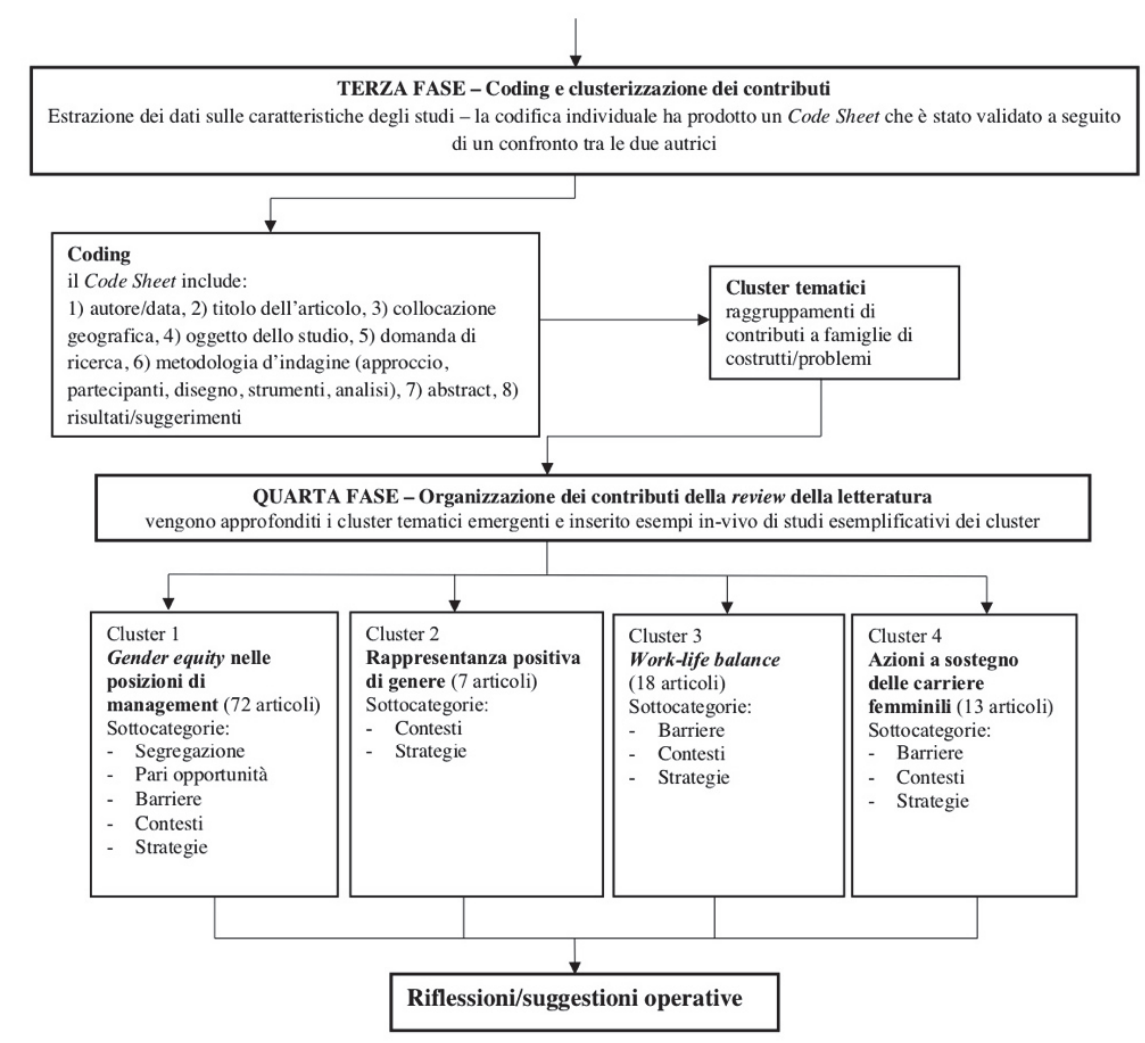

\subsection{IV fase: organizzazione dei contributi della review della letteratura}

Per presentare l'organizzazione dei contributi e la sintesi della review tematica, abbiamo approfondito i cluster concettuali emergenti e inserito esempi invivo.

Si ritiene significativa la suddivisione per paesi/continenti/comunità religiose dei centodieci contributi, attraverso una Literature map (Creswell, 2015) sia visiva (Allegato 1) che descrittiva. I paesi sono riportati in ordine decrescente e il numero degli articoli messo tra parentesi: Italia (34), Usa (19), Australia (13), Regno Unito (4), Asia (3), Canada (3), Cina (3), Europa (3), Germania (3), Croazia (2), India (2), Barbados (1), Belgio (1), Corea (1), Emirati Arabi (1), Danimarca (1), Finlandia (1), Francia (1), Hawaii (1), Irlanda (1), Islam (1), Kenya (1), Malesia (1), Nuova Zelanda (1), Pakistan (1), Portogallo (1), Romania (1), Spagna (1), Sud Africa (2), Tunisia (1), Turchia (1). 


\subsubsection{Cluster tematico 1: gender equity nelle posizioni di management}

Il primo cluster è stato definito "gender equity nelle posizioni di management". Si tratta del cluster più corposo, che include 72 contributi su 110.

È stata osservata la collocazione geografica degli studi presentati riportata in tabella 1.

Tab. 1 - territori che hanno trattato il cluster 1

\begin{tabular}{lcll}
\hline Paese & N. articoli & Paese & N. articoli \\
\hline Italia & 20 & Danimarca & 1 \\
Usa & 15 & Europa & 1 \\
Australia & 9 & Finlandia & 1 \\
Canada & 3 & Francia & 1 \\
Cina & 3 & Hawaii & 1 \\
Asia & 2 & Islam & 1 \\
Germania & 2 & Kenya & 1 \\
India & 2 & Nuova Zelanda & 1 \\
Barbados & 1 & Portogallo & 1 \\
Belgio & 1 & Regno Unito & 1 \\
Corea & 1 & Spagna & 1 \\
Croazia & 1 & Tunisia & 1 \\
\hline
\end{tabular}

Abbiamo intercettato quattro sottocluster che costituiscono i focus tematici delle ricerche analizzate: segregazioni di carriera, pari opportunità, barriere e strategie.

\subsubsection{Segregazioni di carriera}

Le analisi empiriche sulle segregazioni occupazionali verticali esplorano la sottorappresentanza femminile nelle posizioni di top management e di governance, la concentrazione femminile ai livelli più bassi della scala gerarchica organizzativa, e l'esclusione sistematica delle donne da ruoli di leadership nelle aziende. Vi sono poi ricerche sulle segregazioni orizzontali, in cui oggetto di studio sono le discriminazioni materiali nei confronti delle donne, il divario nel trattamento economico e il gap tra uomini e donne nelle imprese. I contesti di ricerca, così come il numero e la tipologia di partecipanti, varia tra uno studio e l'altro. 
Albertini (2011) documenta, in maniera comparativa, la presenza delle donne ai vertici delle maggiori aziende del territorio nazionale. Analizza, in particolare, il grado di femminilizzazione dei consigli di amministrazione e dei vertici della dirigenza di 195 aziende italiane quotate in borsa e inserite nell'indice Ftse Mib. I risultati hanno dimostrato che la proporzione di «donne in vetta» è significativamente più elevata nelle aziende che operano nel settore dei prodotti per la persona, moda, servizi bancari e/o finanziari. La quota di donne è particolarmente bassa ai vertici delle aziende che producono beni industriali e che operano nei settori delle costruzioni, trasporti e produzione di autoveicoli. In altre parole, la rilevanza della presenza femminile ai vertici di una società sembra associata al settore in cui la stessa opera secondo il principio di essenzialismo di genere (gender essentialism); questo principio replica la distorsione culturale secondo cui le donne sono più adatte e competenti nelle attività connesse ai servizi, alle relazioni sociali o alla cura della persona (Charles, 2003).

Uno studio analogo viene descritto da Carbone e Dagnes (2019): hanno realizzato un'indagine tramite web survey tra gennaio e marzo 2017 sull'intera popolazione di donne che al 2015 ricoprivano un incarico in consigli di amministrazione di società quotate presso la borsa valori di Milano. Il numero dei casi analizzati è stato 471 . Nello studio, vengono presentate le azioni positive (o affirmative actions), che costituiscono lo strumento attuativo di politiche antidiscriminatorie a favore di principi di parità. Le azioni positive a favore del riequilibrio di genere nei vertici aziendali si suddividono in due famiglie, a seconda del loro grado di cogenza (Carbone, \& Degnas, 2019):

- le disposizioni soft law - come i codici deontologici delle associazioni di categoria e i codici di autodisciplina adottati dalle imprese - che prevedono raccomandazioni prive di efficacia vincolante, ma che sono invitati a seguire (in alcuni casi con l'obbligo di giustificare l'eventuale inottemperanza, secondo il principio del comply or explain);

- le norme hard law, caratterizzate da obbligatorietà, che assumono la forma di quote garantite al genere meno rappresentato.

Un altro studio rilevante è condotto da Smith (2015) sulle condizioni organizzative che possono facilitare l'accesso a posizioni di leadership da parte delle donne, in particolare nel settore pubblico.

Rifacendosi al costrutto di "scogliera di cristallo", secondo cui le donne accedono a posizioni di governance e di leadership soprattutto quando le organizzazioni attraversano momenti di crisi e sono a rischio di fallimento, la ricerca prende in esame un campione di 911 agenzie educative, distretti scolastici, già notoriamente a forte prevalenza occupazionale femminile.

I risultati della ricerca mostrano che le donne acquisiscono ruoli e posizioni di leadership nelle organizzazioni pubbliche quando queste sono di grandi dimensioni, a prevalenza femminile e a poter decidere chi eleggere sono altre 
donne. In futuro sarà utile indagare in chiave comparativa le traiettorie di carriera di uomini e donne nei distretti scolastici, le possibilità di sviluppo professionale, le culture e pratiche che attraverso processi di socializzazione professionale modellano i percorsi di carriera dei lavoratori e delle lavoratrici (Smith, 2015).

\subsubsection{Pari Opportunità}

Fanno parte del sottocluster delle Pari Opportunità le ricerche che si sono occupate delle politiche adottate all'interno dei consessi amministrativi e manageriali. Si tratta di due macrogruppi:

a. politiche volte a rilevare, denunciare e ridurre la disparità di trattamento tra uomini e donne;

b. politiche volte a contrastare gli stereotipi di genere e a favorire l'accesso a posizioni manageriali da parte delle donne.

Citiamo lo studio comparativo di Strachan, Adikaram e Kailasapathy (2015), che affronta le similarità nelle disparità di trattamento tra uomini e donne in diverse nazioni. Le disuguaglianze più rilevanti e "trans-nazionali" sono: 1'esposizione maggiore delle donne a posizioni lavorative più instabili e precarie; differenze di pagamento; squilibrio nella proporzione tra uomini e donne nelle posizioni manageriali. Le principali barriere allo sviluppo delle carriere di genere sono: la diffusione di credenze stereotipate e distorsioni organizzative, la divisione del lavoro basata sul genere (e non sulle competenze), il conflitto lavoro-famiglia (Kailasapathy, Kraimer, \& Metz, 2014), l'efficacia della normativa a tutela di accessi egualitari al lavoro e la mancanza di un impegno istituzionale volto a garantire un sistema di welfare aziendale. Il lavoro delle Autrici si sofferma soprattutto sui comparti di lavoro delle nazioni del sud-est asiatico, economicamente in crescita ma particolarmente esposte a divari di genere.

\subsubsection{Barriere}

Quali sono le barriere e gli ostacoli all'avanzamento delle carriere femminili nei luoghi di lavoro? Esistono vincoli comuni e trasversali ai diversi contesti organizzativi?

Citiamo a titolo esemplificativo due studi che cercano di sistematizzare e categorizzare le tipologie di barriere occupazionali e di eventuali correttivi messi in atto.

Annabi e Lebovitz (2018) mostrano come malgrado gli sforzi di implementare azioni a sostegno dell'inclusione delle diversità di genere nelle organizzazioni per l'Information Technology (IT), persistano barriere organizzative allo 
sviluppo delle carriere e si rifanno al framework concettuale degli studi sull'information system (IS) (Trauth, 2011). Distinguono nello specifico:

- barriere di tipo organizzativo, quali sistemi di turnover sfavorevoli, assenza di sistemi di ricompensa proporzionali all'impegno verso l'azienda;

- barriere di tipo sociale, quali stress da conflitto lavoro-famiglia, network informali non supportivi, e mancanza di azioni di mentoring.

Soprattutto nel settore delle industrie dell'ICT persistono bias di genere legate ad una visione stereotipicamente mascolina della professionalità e che sottostimano le competenze tecniche del management femminile, malgrado queste favoriscono l'innovazione e la performance dei team ad alta eterogeneità di genere (DuBow, 2013; Ashcraft et al., 2016; Barker, Mancha, \& Ashcraft, 2014). Minore è il numero di donne in posizioni di management, conseguenzialmente minore sarà il supporto di role-modeling e mentoring che altre donne possono ricevere per la propria carriera, più scarni saranno i network professionali (Desvaux et al., 2010). Le Autrici si rifanno all'approccio socioculturale di Trauth (2002), asserendo che sussistono variabilità soggettive nel modo in cui le donne interpretano le barriere occupazionali, e attivano strategie informali per superarle (Trauth, 2002; 2013). L'Individual Differences Theory of Gender and IT (IDTGIT) di Trauth $(2002 ; 2013)$ enuclea tre costrutti interrelati che influenzano la tipologia di risposta individuale che le donne costruiscono:

1. l'identità individuale, in cui la soggettività della persona è definita in funzione delle sue caratteristiche demografiche e dei tratti di carriera;

2. le influenze individuali, vale a dire il complesso intreccio di caratteristiche personali, stili di attribuzione cognitiva e l'adozione di modelli di comportamento socialmente assimilati;

3. le influenze ambientali, che includono le condizioni materiali, economiche, le aspettative comportamentali, le politiche di riferimento e le infrastrutture disponibili.

Nelle industrie dell'Information technology, l'esperienza soggettiva delle barriere presenti è sempre mitigata e filtrata da questi tre livelli di influenze, in cui agiscono metodi di coping individuali e informali e interventi organizzativi volti a strutturare forme di supporto flessibile.

Diehl e Dzubinski (2016) hanno condotto una meta-analisi dei dati prodotti da due studi qualitativi con 38 donne in posizione di leadership. L'analisi ha permesso di rintracciare tre tipologie di barriere allo sviluppo delle carriere femminili:

1. livello macro delle barriere alla leadership, in cui rientrano il controllo dei turni di parola nelle conversazioni, gli stereotipi sui ruoli di genere, l'attribuzione della leadership a caratteristiche di ordine maschile, atteggiamenti di esame ipercritico nei confronti delle donne; 
2. livello meso delle barriere alla leadership, che include il misconoscimento dei contributi delle professioniste, le forme di discriminazione o ostruzionismo velate e implicite, l'esclusione dai network informali, le "scalate di cristallo" con posizionamenti delle professioniste in ruoli di management in fasi di rischio, l'assenza di supporto, disuguaglianze di pagamento e minority distress;

3. livello micro delle barriere alla leadership, tra cui vi sono conflitti lavorofamiglia, sottostima delle proprie abilità, personalizzazione delle difficoltà e delle criticità organizzative, locus of control esterno, vincoli nello stile di comunicazione.

Sul piano nazionale, Villa (2010) analizza l'aumento del tasso occupazionale femminile in Italia nel periodo 1995-2010, correlando il dato al tasso di disoccupazione giovanile, al livello di istruzione, alla localizzazione geografica e all'incidenza degli occupati dipendenti a tempo determinato sul totale per sesso, classe di età e reddito. La "femminilizzazione" del lavoro è effetto dell'incremento della partecipazione delle donne al lavoro retribuito (una delle tendenze maggiormente evidenti negli ultimi trent'anni) e della diffusione della loro presenza nei settori tradizionalmente considerati maschili.

Il costrutto mette in luce il prevalere nei rapporti professionali di specifiche forme di mascolinità, egemoniche e dominanti rispetto alle altre possibili (Collinson, Hearn, 1994). Il concetto di femminilizzazione è stato utilizzato per dar conto della precarizzazione e della progressiva flessibilizzazione del lavoro contemporaneo. Emerge il ritratto di un modello di flex-insecurity per le lavoratrici, laddove l'aumento nel tasso di occupazione femminile non è stato accompagnato né da una riduzione delle difficoltà di inserimento (a parità di caratteristiche il tasso di disoccupazione femminile è sistematicamente più elevato del tasso di disoccupazione maschile), né da un miglioramento della qualità dei lavori offerti alle donne. Pur avendo background formativi brillanti, le professioniste incontrano maggiori difficoltà rispetto ai coetanei nell'accesso a posizioni lavorative più stabili, così come è accentuata la precarizzazione che impatta anche sull'eventuale bilancio lavoro-vita (Villa, 2010).

\subsubsection{Strategie}

Il sottocluster delle azioni e le strategie che sono già in corso per supportare processi di gender equity nelle posizioni di management consente di individuare un repertorio di pratiche e di esperienze consolidate o in fase di consolidamento, che possono traslare anche in setting e contesti differenti.

Vasavada (2012) ha analizzato le caratteristiche attribuite a 32 donne in posizione di governance in organizzazioni profit e no-profit in India. Le donne che ricoprono posizioni di management vengono sottoposte a un processo di 
othering nelle loro organizzazioni, poiché considerate devianti rispetto a una gerarchia e un'opposizione valoriale. Gli uomini possono ricoprire posizioni di comando, le donne no, e se lo fanno, appartengono all'ordine dell'eccezionalità e presentano caratteristiche etero-ascrivibili (stile androgino di comando, equilibrio tra componenti maschili e femminili). Il contributo valorizza le esperienze delle donne che costituiscono una sfida all'androginia obbligatoria e ai suoi confini, adottando una prospettiva culturale femminista e proponendo un processo multidimensionale per accrescere l'accettazione e la valorizzazione della leadership di genere nelle organizzazioni pubbliche e private. Questo modello multidimensionale comprende competenze di coltivazione dei talenti presenti nelle organizzazioni, stili di leadership e livelli di comunicazione interpersonale.

In questo scenario, Terjesen, Sealy e Singh (2009) individuano come strategie sostantive ad ampio raggio: riferimenti legislativi ad hoc, maggior coinvolgimento delle parti sociali e investimento nella sensibilizzazione nei luoghi di lavoro. Intercettano poi alcune strategie a medio raggio, quali:

a. il gender role modeling, laddove donne leader possono ispirare e fare da mentori ad altre donne;

b. diversity supports, dispositivi di facilitazione differenziali e personalizzati, funzionali a supportare carriere con esiti non scontati;

c. gender advocacy e candidature gender-friendly, volte a sostenere l'accesso di tutti a posizioni di governance.

\subsubsection{Cluster tematico 2: rappresentanza positiva di genere}

Il secondo cluster è stato definito "rappresentanza positiva di genere". Pur essendo il cluster con il minor numero di contributi si è ritenuto opportuno inserirlo perché rileva come la presenza di figure femminili, ad alti livelli, possa essere funzionale e positiva per l'azienda.

Un contributo esemplificativo è dato dallo studio condotto da Glass e Cook (2018), che ha indagato l'impatto che le donne, in veste di amministratrici delegate o membri del consiglio di amministrazione, hanno sulle politiche e performance aziendali.

Lo studio si incardina su tre costrutti: la diversità, la differenza di genere e l'omofilia. L'azienda può valorizzare in termini funzionali sia la diversità, generatrice di confronti, problem solving più efficaci e aumento della creatività (van Knippenberg et al., 2004), che la differenza di genere, intesa come traiettorie educative, esperienze lavorative, network professionali differenti tra uomo e donna (Dezsö \& Ross, 2012; Jehn, Northcraft e Neale, 1999). L'omofilia, invece, ha le sue radici nella teoria dell'attrazione secondo cui le persone sono attratte da chi è simile a loro (Ibarra, 1995). Valorizza la presenza di altre donne 
nelle posizioni decisionali, fornendo loro le risorse per fronteggiare i vari ostacoli, come la sfiducia o la mancanza di supporto, creare un clima di solidarietà e produttività.

Le due Autrici rilevano che non è tanto la rappresentanza femminile all'interno dei $\mathrm{CdA}$ a rendere le organizzazioni più solide (sia nella produzione che nelle politiche diversity inclusion) bensì la presenza di donne CEO. L'eterogeneità nei gruppi dirigenziali risulta funzionale al raggiungimento di risultati positivi (Glass, Cook, 2018).

Spostandosi in Romania, Sumendra (2016) ha svolto un'indagine sull'impatto della rappresentanza femminile nei Consigli di amministrazione o nei gruppi dirigenti. Lo studio, pur rilevando una bassa percentuale di donne nei consigli di amministrazione, evidenzia che la presenza femminile nei CdA è propedeutica a rendere partecipi più donne ai processi decisionali, anche se in posizioni di livello intermedio.

In America, già alla fine degli anni '90, all'interno dei Senior Executive Service (SES) - servizi di burocrazia federale - donne e uomini avevano le stesse responsabilità lavorative e anche simili opportunità di sviluppo della propria carriera. Le donne ritenevano di essere più influenti rispetto agli uomini, soprattutto per alcuni aspetti come: consigliare i propri superiori in termini organizzativi, prontezza nell'affrontare questioni e nuovi sviluppi, definire le priorità e proporre possibili modifiche alle normative, politiche o programmi (Dolan, 2004).

In un'ottica legata all'effetto benefico della diversità di genere nei consigli di amministrazione, la ricerca di Capezio e Mavisakalyan (2016), si focalizza sull'impatto della rappresentanza di genere sulle frodi aziendali. Gli autori hanno individuato tre modalità, connesse tra loro ma distinti, che permettono di contenere le frodi:

1) la tendenza, da parte degli uomini, a ostacolare la presenza delle donne nei network importanti che può comportare un'automatica esclusione, delle stesse, dalle culture e dai processi fraudolenti;

2) maggior visibilità delle donne, che produce un effetto positivo sulle loro performance e innalza le loro aspettative di governance;

3) differenza comportamentale, ovvero che le donne sono più efficaci e diligenti nella pratica di monitoraggio.

Attraverso una meta-analisi su dati ottenuti dal Global Banking Fraud Survey nei periodi 2004, 2006 e 2008 sulle frodi in 128 società australiane e neozelandesi quotate in borsa, gli autori dimostrano che l'aumento della presenza delle donne nei consigli mitiga o riduce la presenza di frodi aziendali, sia da parte di dirigenti e impiegati che da soggetti esterni (Capezio, Mavisakalyan, 2016). 
Nella tabella 2 è riportata la provenienza dei 7 contributi inclusi nella nostra review.

Tab. 2 - Territori che hanno trattato il cluster 2

\begin{tabular}{lc} 
Paese & N. articoli \\
\hline USA & 3 \\
Australia & 1 \\
Germania & 1 \\
Regno Unito & 1 \\
Romania & 1 \\
\hline
\end{tabular}

\subsubsection{Cluster tematico 3: work-life balance}

Le modalità della partecipazione femminile al mercato del lavoro sono fortemente correlate all'impatto che le politiche sociali hanno in termini di pari opportunità o di riproduzione delle diseguaglianze sociali (Biancheri, 2006). Il tema della conciliazione tra lavoro e famiglia viene inserito nell'agenda della politica comunitaria come elemento di gender mainstreaming trasversalmente a tutti i settori di intervento. La letteratura dell'ultima decade (2010-2020) sostituisce il costrutto di conciliazione lavoro-famiglia con il costrutto di work-life balance. Si tratta di un'opzione non solo lessicale, ma epistemologica, che restituisce l'attenzione per il benessere di vita della persona lavoratrice, non per la prefigurazione di necessaria conciliazione con una dimensione familiare non scontata.

Abbiamo, quindi, codificato con un label in-vivo il cluster work-life balance. I contributi raggruppati sono 18 e nella tabella 3 si sintetizza la loro provenienza.

Tab. 3 - Territori che hanno trattato il cluster 3

\begin{tabular}{lc} 
Paese & N. articoli \\
\hline Italia & 10 \\
Croazia & 1 \\
Emirati Arabi & 1 \\
Europa & 1 \\
Malesia & 1 \\
Regno Unito & 1 \\
Sud Africa & 1 \\
Turchia & 1 \\
USA & 1 \\
\hline
\end{tabular}


L'analisi di Rizza e Sansavini (2010) sottolinea che la forza normativa delle aspettative istituzionalizzate sulle occupazioni femminili ha contribuito alla definizione del carattere familista del regime di welfare. Questo è contraddistinto da un apparato di regole che attribuisce erroneamente la gestione/cura della filiazione alla componente femminile della genitorialità, generando disattenzione nell'erogazione di servizi pubblici per il sostegno a bambini/anziani e acuendo il conflitto tra lavoro familiare e professionale. In questo quadro, le politiche di conciliazione rappresentano un indicatore dei rapporti di reciproca influenza fra welfare e struttura del mercato del lavoro.

Il risultato è la prevalenza di un modello di conciliazione one-and-half (Rizza, Sansavini, 2010) in cui al lavoro stabile dell'uomo si unisce quello short full-time della donna, svolto in prevalenza all'interno di settori protetti come la burocrazia pubblica o l'insegnamento, nei quali la legislazione garantisce la tutela della maternità e un'agevole fruizione dei congedi. Le due Autrici conducono uno studio di caso multiplo su quattro aziende, operanti nell'industria tessile e nel terziario (servizi alle imprese, servizi alle persone, global service) in Emilia-Romagna. Emerge una genderizzazione delle politiche di conciliazione e bilanciamento tra lavoro e famiglia tutta sbilanciata verso la donna, come se fosse l'unica interessata da strategie di conciliazione. Sono confermati meccanismi di autoselezione che orientano le donne verso determinati settori o forme contrattuali precarie (come part-time e collaborazioni) per consentire loro di farsi carico di obbligazioni intrafamiliari e intergenerazionali. La natura residuale dell'intervento pubblico in tema di servizi alle famiglie favorisce l'insorgere di un senso di abbandono di fronte a sostegni di welfare non adeguati alle richieste sollevate. Sotto questo profilo maternità e paternità, al di là di retoriche pro-natalistiche, sono confinate a dimensioni private e le difficoltà sono vissute dai singoli come un problema personale, familiare e di conseguenza spesso femminile.

È questo il rischio delle politiche di work-life balance, che riproducono trappole di genere orientando su forme di lavoro limitate temporalmente e flessibili che sono dirette alle lavoratrici e non le sostengono nell'avanzamento di carriera.

L'urgenza di predisporre politiche a supporto del work-life balance viene suggerita anche dagli studi che provengono dal Medio Oriente e dal Sud Est Asiatico: invocano politiche di supporto del work-life balance che non siano affidate all'estemporaneità dei singoli casi aziendali, ma formalizzate e istituzionalizzate come agenda di sviluppo sostenibile per le imprese. Forster et al. (2014) si occupano, nello specifico, di come le professioniste degli Emirati Arabi affrontano le richieste competitive del mondo del lavoro e della vita familiare e di quanto queste impattano sulla loro performance professionale e 
sulla percezione di benessere. Hanno somministrato un questionario sulla percezione del work-life balance e del work-family conflict a più di 210 donne impiegate all'interno di compagnie pubbliche e private e hanno selezionato quali tra le rispondenti avessero prole e quali no. I risultati hanno evidenziato che sono le donne che lavorano nelle organizzazioni del settore privato degli Emirati Arabi ad esprimere la richiesta di sostegni per l'equilibrio tra i compiti richiesti dal loro lavoro e la gestione delle attività di vita. L'unica agevolazione riconosciuta sono 45 giorni di congedo per maternità. Le politiche per la sostenibilità del well-being soggettivo attuabili includono: i congedi per paternità non praticati negli Emirati Arabi, lo sviluppo di piattaforme digitali per la consulenza alle aziende che intendono mettere in atto strategie per il work-life balance, e i congedi per maternità distribuiti non solo al momento della nascita ma anche nei primi mesi/anni successivi.

Faisal (2010) ha condotto uno studio qualitativo con 300 donne afferenti ai settori amministrativi, educativi e sanitari del Pakistan. Ha utilizzato l'Indice di Percezione dell'ambiente di lavoro (Perception of Work Environment Index, $P W E I$, Faisal, 2010), che misura il grado di soddisfazione delle donne impiegate nei settori pubblici in rapporto a opportunità di carriera, dispositivi di facilitazione e inter-relazioni a sostegno dell'avanzamento professionale. La versione finale dell'Indice di Percezione dell'ambiente di lavoro comprende tre sottoscale:

- Scala dell'uguaglianza di opportunità per lo sviluppo professionale (Equality of Opportunity for Professional Development Scale, EOPD);

- Scala della protezione e facilitazione del personale femminile (Female Staff Protection and Facilitation, FSPF);

- Scala delle congenialità delle relazioni interpersonali (Congeniality of InterPersonal Relationships, CIPR).

Le partecipanti, pur dichiarando di non percepire il contesto di lavoro come apertamente ostile, non sono pienamente soddisfatte delle condizioni di facilitazione per il work-life balance soprattutto quando sono sposate e con prole. Questo impatta negativamente sull'indice di prevalenza femminile nella governance dei settori pubblici in Pakistan, determinando un effetto di "auto-castrazione" per difficoltà di gestione oppure di "ostilità orizzontale" tra professioniste. Tra le azioni strategiche che lo studio individua (Faisal, 2010) vi sono:

- la costruzione di linee guida per presidiare le politiche e le azioni di gender equity che siano adattate alle caratteristiche delle diverse tipologie di organizzazione, e che non siano generali, quali le facilitazioni per l'assistenza medica e i trasporti; 
- la diffusione capillare delle opportunità di carriera nell'organizzazione e l'incoraggiamento alle donne ad accedervi, fornendo, ad esempio, possibilità di trasporto per chi partecipa a percorsi formativi e aprendo servizi per l'infanzia sino a tardi;

- la sensibilizzazione di genere attraverso percorsi di sviluppo professionale che siano fondati su metodologie per la riflessione e l'analisi critica delle pratiche distorte in azienda;

- il supporto attivo alla professionalizzazione delle donne e all'acquisizione di titoli culturali altamente qualificati, come specializzazioni e percorsi dottorali.

\subsubsection{Cluster tematico 4: azioni a sostegno delle carriere femminili}

I 13 contributi che sono stati raggruppati sotto questo cluster presentano esempi di azioni a sostegno delle carriere femminili; nella tabella 4 ne viene rappresentata la provenienza.

\begin{tabular}{lc} 
Tab. 4 - Territori che hanno trattato il cluster 4 & \\
\hline Paese & N. articoli \\
\hline Italia & 4 \\
Australia & 3 \\
Asia & 1 \\
Irlanda & 1 \\
Pakistan & 1 \\
Regno Unito & 1 \\
Sud Africa & 1 \\
Usa & 1 \\
\hline
\end{tabular}

Riportiamo due contributi esemplificativi che approfondiscono l'impatto di percorsi di sviluppo professionale. In Australia, il contributo di Clarke (2011) tratta del programma HIWL - High Impact Women in Leadership, rivolto a donne manager o che evidenziano una spiccata capacità di leadership. Il percorso, attraverso moduli e attività interattive (workshop, case-study, esperienze di testimoni privilegiati, coaching), permette di potenziare le competenze utili a un possibile avanzamento di carriera. In Irlanda, Potter e Hill (2009) analizzano il progetto WINS (Women into Non-traditional Sectors), sviluppato in diverse fasi (reclutamento, supporto, formazione), che dava l'occasione alle donne di lavorare in settori non tradizionali (es. autiste di autobus, guardie di quartiere e nel settore dell'edilizia) prevalentemente popolati da uomini. Anche 
se entrambi avevano come focus il sostegno alle carriere femminili, il primo era relativo alla de-segregazione verticale mentre il secondo a quella orizzontale.

Ridurre il gender gap oggi richiede non solo di attivare percorsi rivolti alle donne, ma anche di investire sulla promozione di una differente cultura organizzativa e sull'attuazione di pratiche di gender equity.

Lo studio di Annabi e Lebovitz (2018), citato precedentemente, valuta la percezione dell'efficacia di metodi di supporto allo sviluppo delle carriere femminili nei settori dell'Information Technology. Le pratiche emerse dal loro contributo sono:

1. costruire culture organizzative che valorizzino le performance di team ad alta variabilità di genere;

2. fornire arrangiamenti flessibili per mitigare gli effetti del work-family conflict e strutture di lavoro agile;

3. attuare percorsi di apprendimento dall'esperienza che consentano di esaminare le pratiche velatamente discriminatorie;

4. migliorare le azioni di coaching e mentoring con donne manager e leader;

5. offrire opportunità di sviluppo professionale con programmi di leadership e di rafforzamento delle competenze tecniche (Annabi, Lebovitz, 2018).

Civita, Pietropaolo, Montaruli (2013) esaminano l'impatto del progetto M.A.F.A.L.D.A. - Misure a Favore di Azioni per il Lavoro, i Diritti e le Affettività nel creare un network tra istituzioni pubbliche e organizzazioni private $\mathrm{e}$ sviluppare attività che coinvolgessero tutta la cittadinanza. Le azioni intraprese sono state (Civita, Pietropaolo, Montaruli, 2013):

1. incentivare le organizzazioni ad attuare pratiche work-life balance;

2. creazione di un sistema informativo e di banche dati contenenti indicatori di genere;

3. eventi informativi sull'uguaglianza di genere negli ambiti familiare, lavorativo e sociale;

4. promozione del progetto e attuazione di laboratori di sensibilizzazione nelle scuole secondarie di I grado;

5. attività di orientamento al lavoro e all'autoimprenditorialità;

6. supporto formativo e pratico, che ha visto coinvolte venti donne disoccupate o inoccupate, per la creazione di una start-up, la cooperativa Tagesmutter (mamme di giorno).

Infine Debebe, Anderson, Bilimoria, e Vinnicombe (2016) analizzano cinque sfide chiave dei programmi di leadership a sostegno delle carriere delle donne. Il loro framework teorico incrocia i costrutti di apprendimento trasformativo (Mezirow, 2000), cambiamento organizzativo e intersezionalità (Crenshaw, 2017). Il focus è comprendere come i programmi di sviluppo della leadership delle donne possano essere uno strumento per facilitare lo sviluppo di una leadership mindset nelle donne. I temi analizzati sono: 
1. la composizione di genere nei programmi di leadership;

2. l'adozione di prospettive intersezionali nei programmi di leadership, che non normalizzino l'esperienza di donne bianche o visioni binarie dei generi;

3. la dimensione collaborativa della costruzione dei programmi di leadership, in cui adottare i costrutti di apprendimento trasformativo, di autostima, e autoefficacia;

4. la valutazione dei risultati dei programmi di leadership, che includono metodologie autoetnografiche dove piccoli gruppi di donne sono coinvolte in attività di peer coaching, in metodologie dialogiche per la riflessione critica e in attività di coaching personalizzato;

5. l'embedding dei programmi di leadership femminile nelle organizzazioni, progettando i programmi direttamente con le partecipanti e gli attori organizzativi apicali, e conducendo percorsi di formazione on-the-job.

Le best practices di questi ultimi tre contributi sono riportate in tabella 5.

Tab. 5 - Best practices a sostegno delle carriere femminili

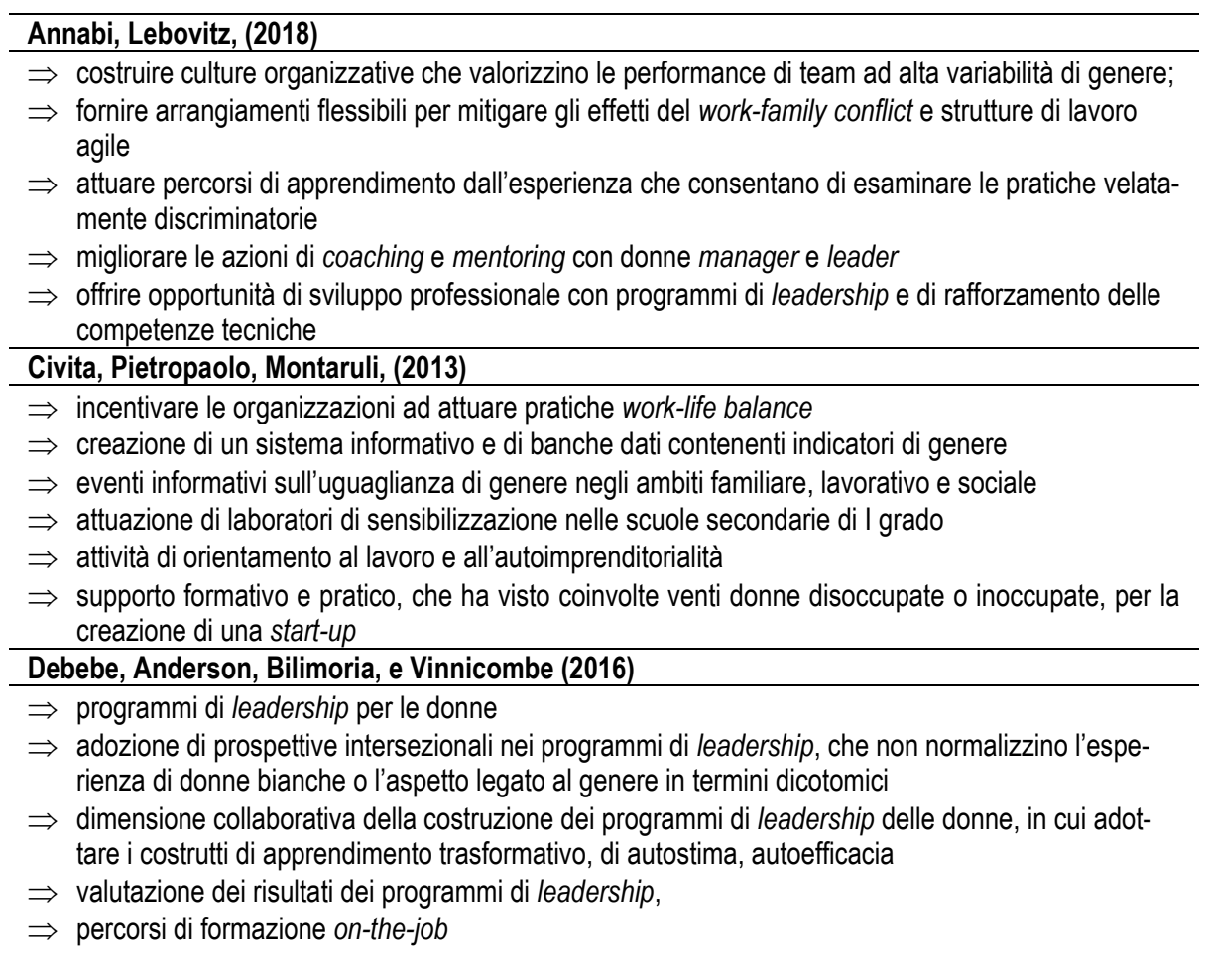




\section{Tra gender knowledge e inclusive management}

La review sistematica della letteratura costituisce un primo dispositivo di facilitazione per esplorare e implementare il corpo di conoscenze sui temi del gender diversity management e il sostegno alle carriere femminili. Distinguiamo le implicazioni scientifiche e le implicazioni organizzative del contributo:

- con implicazioni scientifiche intendiamo il lavoro di spacchettamento dei costrutti di gender equity, work-life balance e gender management nelle organizzazioni, la ricostruzione del quadro delle ricerche a livello internazionale e nazionale;

- con implicazioni organizzative intendiamo l'emergenza di pratiche e dispositivi a sostegno del work-life balance e delle carriere femminili, e di strumenti di intervento a favore della gender equity e della leadership di genere che possono essere adottati e traslare nelle diverse realtà organizzative.

Si tratta di pratiche che testimoniano di processi di cambiamento organizzativo e di innovazione sostenibile verso cui si stanno dirigendo le organizzazioni che vogliono investire nella valorizzazione del capitale umano e nella corporate social responsibility.

Dal punto di vista scientifico, future traiettorie di sviluppo saranno l'incremento della letteratura nazionale di settore educativo dedicata ai temi del gender diversity management. Prevalgono, difatti, i contributi che derivano da settori degli studi organizzativi, manageriali e imprenditoriali.

A fronte di questo quadro, produrre conoscenze utili al cambiamento delle culture organizzative è una sfida per lo stesso processo di civilizzazione inclusiva della società.

Le organizzazioni e i workplace, a differenza di quanto dichiarano, non sono neutri, ma sono strutturati secondo il simbolismo del genere e la loro cultura è "gendered". Gli studi sulle carriere femminili nel mondo del lavoro sono un laboratorio per le negoziazioni quotidiane delle relazioni tra generi e di generi. Costituiscono osservatori scientifici a partire dai quali organizzare pluralità di dispositivi che non concepiscano ruoli in posizioni subalterne. In questo senso, contribuiscono a "fare" genere, a costruirlo discorsivamente, a sfidare l'ordine simbolico dominante patriarcale per aprire la strada a pratiche che siano più inclusive, gender-sensitive ed emancipanti.

Al contempo, i contesti studiati risultano limitati, come le connessioni teoriche ai costrutti di intersezionalità (see Crenshaw, 2017). L'adozione di prospettive intersezionali consente di riconoscere che le esperienze di carriera delle donne non sono influenzate solo dal genere, ma anche dall'etnia, dalla sessualità, dalla nazionalità, dalla religione, dallo status sociale. Sono influenzate poi dal contesto organizzativo, inteso sia come la cultura dell'organizzazione sia 
come le più specifiche culture di team e di gruppi di appartenenza. Questo significa che le carriere delle donne attraversano scenari incerti, ambigui, paludosi, dove stagnano situazioni paradossali in cui possono incontrare supporti e vincoli allo stesso tempo. Le prospettive intersezionali, in questo senso, offrono uno sguardo d'insieme e meno dicotomico e binario: permettono di rilevare l'intersezione delle dimensioni di doppia o tripla subordinazione culturale che possono vivere nei contesti di lavoro (Debebe, Anderson, Bilimoria \& Vinnicombe, 2016).

Concludiamo condividendo il valore emancipativo di pratiche educative che orientino a prefigurazioni professionali libere da stereotipi di genere fin dal percorso scolastico e universitario. La letteratura, difatti, ci restituisce che è proprio nell'ambito del percorso formativo che studenti/esse acquisiscono, attraverso i processi di socializzazione tra pari e gli apprendimenti formali e informali, le prime aspettative gender-based su ciò che faranno e che potrebbero fare. In aggiunta all'incremento di pratiche di gender diversity management nelle organizzazioni, è promettente la sperimentazione di laboratori e dispositivi di orientamento di genere al lavoro, fin dalle scuole superiori e durante il percorso universitario, che supportino la costruzione di prefigurazioni di carriera sfidanti (Bianchi, Fabbri \& Romano, 2018). Tutto ciò al fine di promuovere percorsi di inclusione di genere all'interno dei sistemi culturali e organizzativi e nell'interesse di praticare esperienze funzionali a prefigurare parabole professionali di genere qualitativamente differenti.

\section{Bibliografia}

Agyemang-Mintah P., \& Schadewitz H. (2019). Gender diversity and firm value: evidence from UK Financial Institutions. International Journal of Accounting \& Information Management, 27: 1: 2-26. doi:10.1108/IJAIM-06-2017-0073.

Ahuja M. (2002). Women in the information technology profession: A literature review, synthesis, and research agenda. European Journal of Information Systems, 11: 20-34. doi: $10.1057 /$ palgrave.ejis.3000417.

Albertini M. (2011). Donne in vetta. Il soffitto di cristallo nelle grandi aziende italiane in prospettiva comparata. Polis, 25: 3. doi:10.1424/35846.

Annabi H. \& Lebovitz S. (2018). Improving the retention of women in the IT workforce: an investigation of gender diversity interventions in the USA. Information Systems Journal, 28(6): 1049-1082. doi:10.1111/isj.12182.

Ashcraft C., McLain B., \& Eger E. (2016). Women in Tech: The facts. Boulder, CO: National Center for Women and Information Technology.

Barker L., Mancha C., \& Ashcraft C. (2014). What is the impact of gender diversity on technology business performance? Research summary. Boulder, CO: National Center for Women and Information Technology. 
Biancheri R. (2006). Mercato del lavoro, famiglia e politiche sociali: un'analisi sociologica delle carriere femminili. Lavoro $e$ diritto, 20(4): 581-610. doi: $10.1441 / 22711$.

Blackburn R.M., et al. (2002). Explaining Gender Segregation. The British Journal of Sociology, 53(4): 513-536. doi: 10.1080/0007131022000021461.

Capezio Al., \& Mavisakalyan A. (2016). Women in the boardroom and fraud: evidence from Australia. Australian Journal of Management, 41(4): 719-734. doi: 10.1177/0312896215579463.

Carbone D., Dagnes J. (2019). Fare carriera ai vertici. La presenza delle donne nei consigli di amministrazione tra vecchi ostacoli e nuove opportunità. Polis, 33(2): doi: $10.1424 / 94249$.

Charles M. (2003) Deciphering Sex Segregation. Vertical and Horizontal Inequalities in Ten National Labor Markets. Acta Sociologica, 46(4): 267-287. doi: 10.1177/0001699303464001.

Civita A., Pietropaolo L. \& Montarulli D. (2013). Disuguaglianza di genere e Politiche di intervento in Puglia. Autonomie Locali e Servizi Sociali, 2. doi: 10.1447/75375.

Clarke M. (2011). Advancing Women's Careers through Leadership Development Programs. Employee Relations, 33(5): 498-515. doi: 10.1108/01425451111153871.

Crenshaw K. (2017). On intersectionality: essential writings. New York: The New Press.

Collinson D., \& Hearn J. (1994). Naming men as men: Implications for work, organization and management. Gender, Work and Organization, 1: 2-22.

Creswell J.W. \& Guettermann T. C. (2015). Educational research: planning, conducting, and evaluating quantitative and qualitative research (Sixth Edition). Boston, MA: Pearson Edition.

Debebe G., Anderson D., Bilimoria D. \& Vinnicombe S.M. (2016). Women's leadership development programs: lessons learned and new frontiers. Journal of Management Education, 40(3): 231-252. doi: 10.1177/1052562916639079.

Denyer D., \& Tranfield D. (2009). Producing a systematic review. In Buchanan D. and Bryman A. (eds). The Sage Handbook of Organizational Research Methods. London: Sage, pp. 671-689.

Desvaux G., Devillard S., \& Sancier-Sultan S. (2010). Women at the top of corporations: making it happen. McKinsey \& Company (http://www.mckinsey.com/ /me$\mathrm{dia} / \mathrm{mckinsey} /$ dotcom/client_service/Organization/PDFs/Women_matter_oct2010_english.ashx). Retrieved October 1, 2011.

Devicienti F., Grinza E., Manello A., \& Vannoni D. (2019). What are the benefits of having more female leaders? Evidence from the use of part-time work in Italy. Industrial and Labor Relations Review, 72(4): 897-926. doi: 10.1177/0019793918800287.

Dezsö C., \& Ross D. G. (2012). Does female representation in top management improve firm performance? A panel data investigation. Strategic Management Journal, 33: 1072-1089. doi: 10.1002/smj.1955.

Diehl A.B. \& Dzubinski L.M. (2016). Making the invisible visible: a cross-sector analysis of gender-based leadership barriers. Human Resource Development Quarterly, 27(2): 181-206. doi: 10.1002/hrdq.21248. 
Dolan J. (2004). Gender equity: illusion or reality for women in the Federal Executive Service?. Public Administration Review, 64(3): 299-309. doi: 10.1111/j.15406210.2004.00374.x.

DuBow W. M. (2013). Diversity in computing: why it matters and how organizations can achieve it. Computer, 46(3): 24-29. doi: 10.1109/MC.2013.6.

Faisal F. (2010). Measuring perceptions of work environment among educated female public servants in Pakistan. Pakistan Economic and Social Review Volume, 48(1): 135-165.

Feak C. B. \& Swales J. M. (2009). Telling a research story: writing a literature review. University of Michigan Press.

Forster N., Al Ali Ebrahim N. \& Ibrahim N.A. (2014). An exploratory study of worklife balance and work-family conflicts in the United Arab Emirates. Skyline Business Journal, 9(1): 34-42.

Fraser K. D. \& al Sayah F. (2011). Arts-based methods in health research: a systematic review of the literature, Arts \& Health, 3(2): 110-145. doi: 10.1080/17533015.2011.561357.

Glass C., Cook A. (2018). Do women leaders promote positive change? Analyzing the effect of gender on business practices and diversity initiatives. Human Resource Management, 57(4): 823-838. doi: 10.1002/hrm.21838.

Ibarra H. (1995). Race, opportunity, and diversity of social circles in managerial networks. Academy of Management Journal, 38(3): 673-703. doi: 10.5465/256742.

Jehn K., Northcraft G., \& Neale M. (1999). Why differences make a difference: a field study of diversity, conflict and performance in work- groups. Administrative Science Quarterly, 44(4): 741-763. doi: 10.2307/2667054.

Jones O., \& Gatrell C. (2014). Editorial: the future of writing and reviewing for IJMR. International Journal of Management Reviews, 16: 249-264. doi: 10.1111/ijmr.12038.

Kailasapathy P., Kraimer M., \& Metz I. (2014). The interactive effects of leader-member exchange, gender, and spouse's gender role orientation on work interference with family conflict. International Journal of Human Resource Management, 25(19): 2681-2701. doi: 10.1080/09585192.2014.891637.

Mezirow J. (Ed.) (2000). Learning as transformation: Critical perspectives on a theory in progress. San Francisco, CA: Jossey-Bass.

Nix N., \& Stiffler D. (2016). Women in Supply Chain. Supply Chain Management Review, 20(5): 44-51.

Nolan C.T., \& Garavan T.N. (2016). Human resource development in SMEs: a systematic review of the literature. International Journal of Management Reviews, 18: 85107. doi: 10.1111/ijmr.12062.

Özbilgin M.F., Beauregard T.A., Tatli A., \& Bell M.P. (2011). Work-life, diversity and intersectionality: a critical review and research agenda. International Journal of Management Reviews, 13: 177-198. doi: 10.1111/j.1468-2370.2010.00291.x.

Potter M., \& Hill M. (2009). Women into non-traditional sectors: addressing gender segregation in the Northern Ireland workplace. Journal of Vocational Education \& Training, 61(2): 133-150. doi: 10.1080/13636820902933239. 
Rizza R. \& Sansavini M. (2010). Donne e lavoro: rappresentazioni del femminile e conseguenze in termini di politiche di "work-life balance". Rassegna Italiana di Sociologia, 51(1): 5-31. doi: 10.1423/31729.

Romano A., Fabbri L., \& Bianchi F. (2018). "Scusate se voglio far carriera": pratiche trasformative per l'educazione di genere. In: A. Murgia, B. Poggio (a cura di). Saperi di genere. Prospettive interdisciplinari su formazione, lavoro, politiche e movimenti sociali. GARCIA (EU $7^{\circ}$ Framework Programme), Trento, Università di Trento, PP. 104-123.

Samek Lodovici M., \& Semenza R. (2007). Il caso italiano: dalla regolazione dei contratti alla riforma del welfare? La Rivista delle Politiche Sociali, 2: 107-130.

Smith A.E. (2015). On the edge of a glass cliff: women in leadership in public organizations. Public Administration Quarterly, 39(3): 484-518.

Strachan G., Adikaram A., \& Kailasapathy P. (2015). Gender (in)equality in South Asia: problems, prospects and pathways. South Asian Journal of Human Resources Management, 2(1): 1-11. doi: 10.1177/2322093715580222.

Stumbitz B., Lewis S., \& Rouse J. (2018). Maternity management in SMEs: a transdisciplinary review and research agenda. International Journal of Management Reviews, 20: 500-522. DOI: 10.1111/ijmr.12143.

Sumedrea S. (2016). Gender diversity and firm performance in seeking for sustainable development. Bulletin of the Transilvania University of Brasov. Series V: Economic Sciences, 9(58): 2: 369-385.

Taylor E. W. (2017). Critical reflection and transformative learning: a critical review. PAACE Journal of Lifelong Learning, 26(2): 77-95.

Terjesen S., Sealy R. \& Singh V. (2009). Women directors on corporate boards: a review and research agenda. Corporate Governance: An International Review, 17(3): 320-337. doi: 10.1111/j.1467-8683.2009.00742.x.

Trauth E. (2002). Odd girl out: An individual differences perspective on women in the IT profession. Information Technology and People, 15(2): 98-118. doi: $10.1108 / 09593840210430552$.

Trauth E. (2011). Rethinking gender and MIS for the twenty-first century. In R. Galliers, \& W. Currie (Eds.). The Oxford Handbook on MIS. Oxford: Oxford University Press. doi: 10.1093/oxfordhb/9780199580583.003.0025.

Trauth E. (2013). The role of theory in gender and information systems research. Information \& Organization, 23: 277-293. doi: 10.1016/j.infoandorg.2013.08.003.

van Knippenberg D., De Dreu C.K.W., \& Homan A.C. (2004). Work group diversity and group performance: an integrative model and research agenda. Journal of Applied Psychology, 89: 1008-1022.

Vasavada T. (2012). A cultural feminist perspective on leadership in nonprofit organizations: a case of women leaders in India. Public Administration Quarterly, 36(4): 462-503.

Villa P. (2010). La crescita dell'occupazione femminile: la polarizzazione tra stabilità e precarietà. Lavoro e diritto, 25(3): 343-358. doi: 10.1441/32521.

Wang C.L., \& Chugh H. (2014). Entrepreneurial learning: past research and future challenges. International Journal of Management Reviews, 16: 24-61. doi: 10.1111/ijmr.12007. 


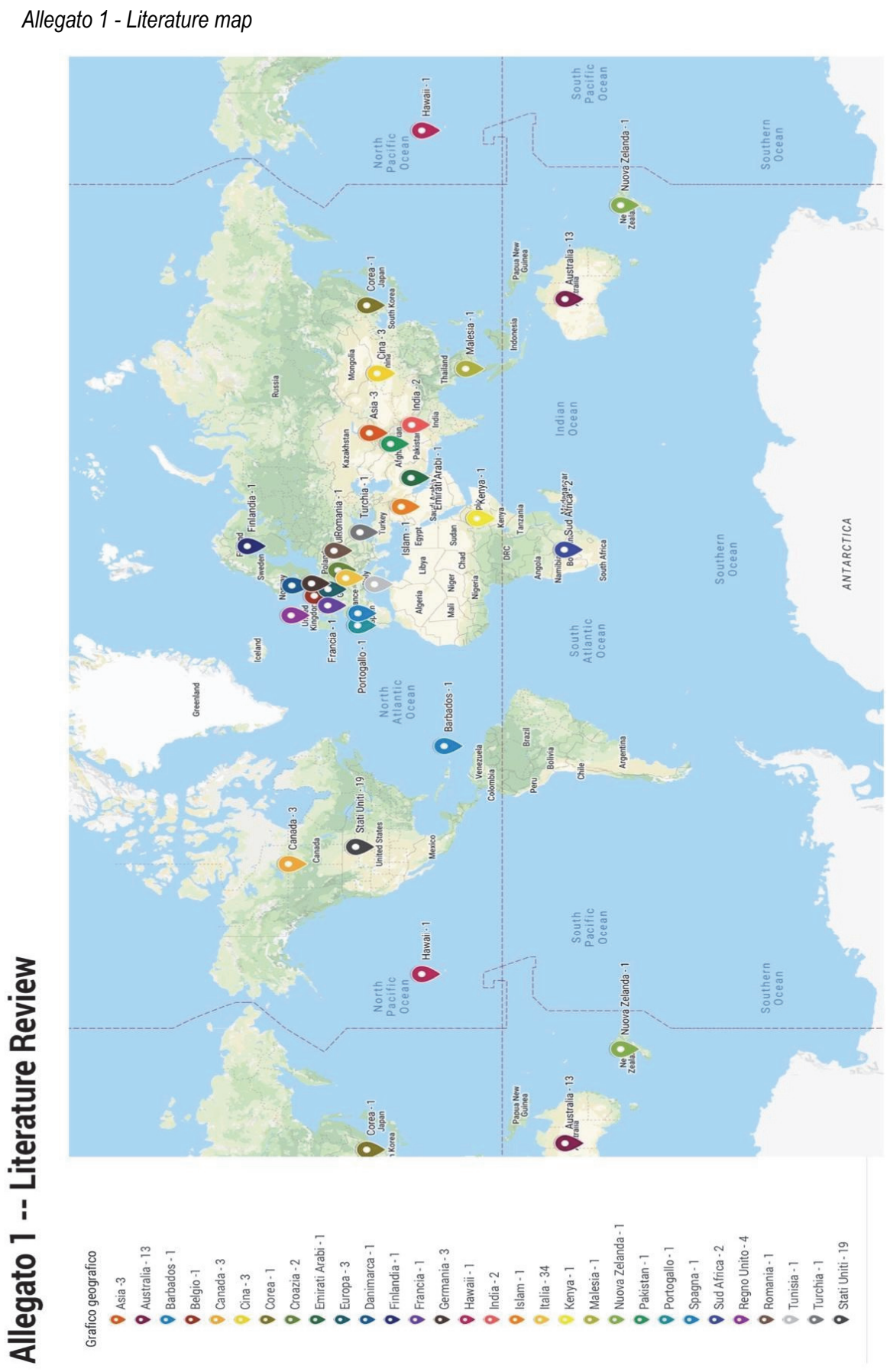

